\title{
Emulating Wired Backhaul with Wireless Network Coding
}

\author{
Thomsen, Henning; De Carvalho, Elisabeth; Popovski, Petar
}

Published in:

General Assembly and Scientific Symposium (URSI GASS), 2014 XXXIth URSI

DOI (link to publication from Publisher):

10.1109/URSIGASS.2014.6929289

Publication date:

2014

\section{Document Version}

Accepted author manuscript, peer reviewed version

Link to publication from Aalborg University

Citation for published version (APA):

Thomsen, H., De Carvalho, E., \& Popovski, P. (2014). Emulating Wired Backhaul with Wireless Network Coding. In General Assembly and Scientific Symposium (URSI GASS), 2014 XXXIth URSI (pp. 1-4). [2950] IEEE Press. General Assembly and Scientific Symposium (URSI GASS) https://doi.org/10.1109/URSIGASS.2014.6929289

\section{General rights}

Copyright and moral rights for the publications made accessible in the public portal are retained by the authors and/or other copyright owners and it is a condition of accessing publications that users recognise and abide by the legal requirements associated with these rights.

- Users may download and print one copy of any publication from the public portal for the purpose of private study or research.

- You may not further distribute the material or use it for any profit-making activity or commercial gain

- You may freely distribute the URL identifying the publication in the public portal -

\section{Take down policy}

If you believe that this document breaches copyright please contact us at vbn@aub.aau.dk providing details, and we will remove access to the work immediately and investigate your claim. 


\title{
Emulating Wired Backhaul with Wireless Network Coding
}

\author{
Henning Thomsen*, Elisabeth De Carvalho, Petar Popovski \\ Department of Electronic Systems, Aalborg University \\ Frederik Bajers Vej 7, DK-9220 Aalborg East, Denmark \\ E-mail: $\{$ ht,edc,petarp\}@es.aau.dk
}

\section{Abstract}

In this paper we address the need for wireless network densification. We propose a solution wherein the wired backhaul employed in heterogeneous cellular networks is replaced with wireless links, while maintaining the rate requirements of the uplink and downlink traffic of each user. The first component of our solution consists of a two-way, two-phase communication between the macro base station and a user in a small cell through the small cell base station. The second component consists of an optimized adjustment of the transmit power from the macro base station during the multiple access phase of the two-way protocol. The transmit power is set high enough to enable successive decoding at the small cell base station where the downlink data to the user is first decoded and its contribution removed from the received signal followed by the uplink data from the user. The decoding of the second layer, the uplink traffic to the user, remains identical to the one performed in a wired system. In the broadcast phase, the decoding of the downlink traffic can also be guaranteed to remain identical. Hence, our solution claims an emulation of a wired backhaul with wireless network coding with same performance. We provide numerical examples involving a macro base stations serving a single small cell or two small cells.

\section{Motivation and Introduction}

There is a growing evidence that small cells will play a major role in the upcoming generation of wireless communication systems [1]. This is in line with the trend of wireless network densification [2], which indicates that the bit rate per unit area will grow immensely. The key element in small cell deployments is the backhaul connection that connects the small cell Base Stations (SBSs) to the infrastructure. The choice of the backhaul needs to hit the right tradeoff between the connectivity and deployment flexibility/cost, thus putting into consideration a mix of wired and wireless backhaul solutions.

Fig. 1 shows an example deployment of three small cells within the coverage area of the macrocell. Each Small Base Station (SBS) uses a wired backhaul to connect to the common infrastructure. We consider a Time-Division Duplex (TDD) operation and each Mobile Station (MS) associated with one SBS uses equal periods, each of duration $T$, to receive the downlink traffic from the SBS and transmit the uplink traffic to the SBS. Specifically, the downlink and uplink data rate of the $k$-th user terminal is denoted by $R_{D, k}$ and $R_{U, k}$, respectively. When there is no danger of causing confusion, we will drop the index $k$ and use simply $R_{D}$ and $R_{U}$. The capacity of the backhaul link is denoted by $C_{B}$ and it is assumed that:

$$
C_{B} \geq \max \left\{R_{D}, R_{U}\right\} .
$$

Two observations are in order. First, even if the wired backhaul is capable of full-duplex transmission, it is essentially used in a TDD manner, as dictated by the TDD regime in the wireless access part, from SBS to the terminal. This means that, at a given instant, the data flow over the wire can be considered to be unidirectional. Second, the capacity $C_{B}$ of the wire does not need to be excessively high. It needs to be just above the uplink/downlink rates that can be supported by the wireless access part, in order for (1) to be satisfied. If multiple users are present in the small cells, then $R_{D}$ and $R_{U}$ in (1) represent the sum-rates of all users.

The central question treated in this paper is the following: How to remove the wired backhaul and rely on wireless connections between BS and the SBSs, while preserving the same performance of the two-way communication with a Mobile Station connected to the small cell?

The motivation for doing that is the need for network densification and deployment of SBSs that is rapid, flexible and low cost. We will refer to the solution of the above problem as a Wireless-Emulated Wire (WEW), since we would like to preserve the uplink/downlink rates of the user to be the same as if the wired backhaul is present. The main assumption is that the wireless backhaul BS-SBS link uses the same spectrum as the link SBS-MS, which implies that SBS has the role of a relay. WEW is possible only when two-way traffic is considered from the MS.

The operation of WEW is based on two principles: First, by having both BS and a MS transmitting to each SBS simultaneously, we create a two-way communication flow between them. This is enabled by the principle of network 


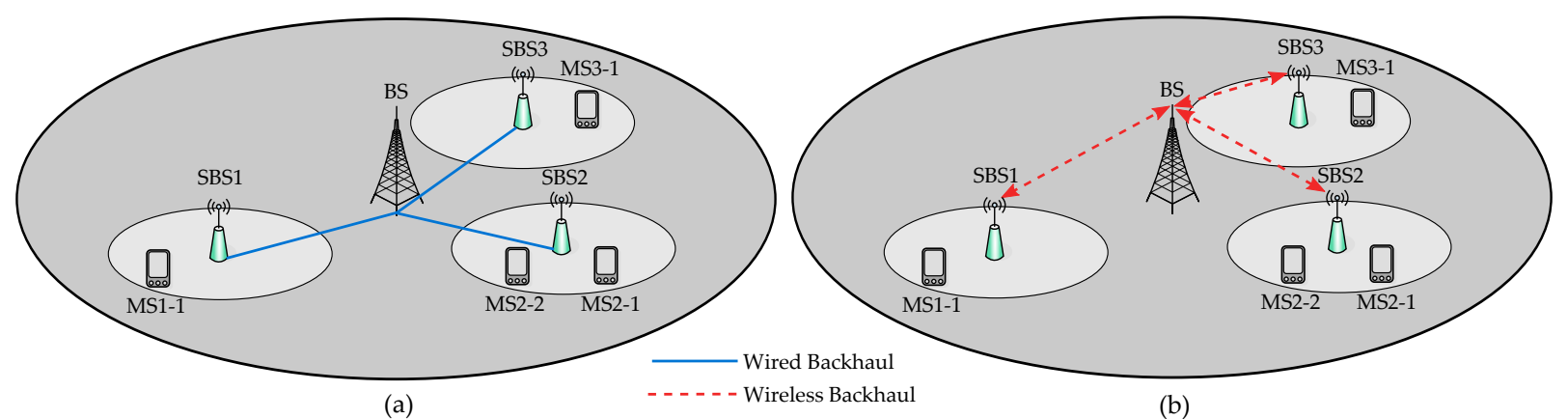

Figure 1: (a) Original deployment with wired backhauls (b) Deployment where the wired backhaul is emulated by the wireless backhaul.

coding [4], [5]. Second, each transmitting node knows its own signal a priori, and can therefore subtract it from any received signal. Previously, these principles have been applied in [6], [7] in the study of coordinated transmissions in a cellular network. We use these principles in creating a wireless backhaul solution.

\section{System Model}

We consider a cellular scenario consisting of one BS and several SBSs. Each SBS serves a number of MSs in its small cell. The case of one BS and three SBSs is shown in Figure 1(b). All MSs are assumed to have two-way, infinitely backlogged traffic to the BS. In our model, every node is assumed to have full Channel State Information (CSI). The uplink rate requirement of $\mathrm{MSi}-\mathrm{j}$ is $R_{U i j}$ bps, while it has a downlink rate requirement of $R_{D i j}$ bps. All nodes are assumed to operate in half-duplex mode. Without loss of generality, we normalize the bandwidth to $1 \mathrm{~Hz}$. Since we impose that WEW needs to be transparent to the MSs, their transmission power is assumed to be the same as the wired backhaul case, as is the channel between each MS and its SBS. Each SBS has a fixed transmission power identical to the wired backhaul case. In order for that to work, we need to assume that the channel SBS-BS is better than the channel SBS-MS. WIth that, SBS can broadcast XOR-ed data to both BS and MS at the rate of the SBS-MS link, which is identical to the downlink rate in the wired backhaul. Our goal is thus to find the minimal transmission power at the BS, such that the rate requirements of the MSs can be fulfilled.

The uplink and downlink transmissions are done over two phases. In the first phase (Multiple Access (MA) phase), the BS and MSi-j transmit simultaneously to SBSi. The Multiple Access Channel (MAC) rate region at each SBSi consists of all uplink and downlink rates that are achievable at this SBSi, and depends on the transmission power at the BS and MS. Since each MS transmits at rate equal to capacity of the link MS-SBS, each SBS is required to decode all received signals, such that th uplink signal from the MS is decoded last, in absence of any interference and thus under identical conditions as in the wired case. In the second phase (the Broadcast Channel (BC) phase), the SBS transmits the exclusive-or (XOR) of the decoded signals in the first phase. The BS and each MS then receive this signal, and because each node knows its own transmitted signal (has side information), it can decode the XORed signal to obtain the desired signal.

\section{WEW with one SBS and one MS}

We first consider the case of one MS, one SBS and one BS. The channel between MS and SBS has capacity $C\left(\gamma_{M}\right)=$ $\log _{2}\left(1+\gamma_{M}\right)$, where $\gamma_{M}=\frac{P_{M}\left|h_{M}\right|^{2}}{\sigma^{2}}$ is the SNR between MS and SBS, $P_{M}$ is the transmit power of the MS, and $\sigma^{2}$ the power of the additive white Gaussian noise. This channel is assumed to be able to support the rates $R_{D}$ and $R_{U}$, i.e. $R_{D}, R_{U} \leq C\left(1+\gamma_{M}\right)$. For the SBS-BS link, we need to find the $P_{B}$ that supports the rate $R_{D}$. At the SBS, we have a MAC with rate bounds

$$
\begin{aligned}
R_{U} & \leq C\left(\gamma_{M}\right), \\
R_{D} & \leq C\left(\gamma_{B}\right), \\
R_{U}+R_{D} & \leq C\left(\gamma_{M}+\gamma_{B}\right) .
\end{aligned}
$$


By assumption, MS transmits at rate equal to capacity, so $R_{U}=C\left(\gamma_{M}\right)$. Then, we need to solve the third inequality for $P_{B}$, to find the condition on the transmission power. We have

$$
\begin{gathered}
\log _{2}\left(1+\gamma_{M}\right)+R_{D} \leq \log _{2}\left(1+\gamma_{M}+\gamma_{D}\right) \Leftrightarrow \\
\log _{2}\left(1+\frac{P_{M}\left|h_{M}\right|^{2}}{\sigma^{2}}\right)+R_{D} \leq \log _{2}\left(1+\frac{P_{M}\left|h_{M}\right|^{2}}{\sigma^{2}}+\frac{P_{B}\left|h_{B}\right|^{2}}{\sigma^{2}}\right) \Leftrightarrow \\
\frac{\sigma^{2}}{\left|h_{B}\right|^{2}}\left(1+\frac{P_{M}\left|h_{M}\right|^{2}}{\sigma^{2}}\right)\left(2^{R_{D}}-1\right) \leq P_{B}
\end{gathered}
$$

which is the condition on the transmission power at the BS, $P_{B}$, in order for the SBS-BS link to support the required rates. The feasibility of WEW for the second phase follows from the assumption that the MS-SBS channel is identical to the wired backhaul case, and from Eq (1).

\section{WEW with two SBSs and two MSs}

To illustrate the case of two small cells, we consider a scenario consisting of one BS, two small BSs SBS1 and SBS2, each serving one MS. The channel between SBSi and its MS is $h_{M i} \in \mathbb{C}$, while the channel between BS and SBSi is $\boldsymbol{h}_{B i} \in \mathbb{C}^{[2 M \times 1]}, M$ being the number of antennas at the BS. The BS uses zero forcing to create spatially separated channels [3], and $\boldsymbol{w}_{B i} \in \mathbb{C}^{[2 M \times 1]}$ is the beam forming vector for SBSi. The downlink signal for MSi is $x_{B i} \in \mathbb{C}$, which is transmitted with power $P_{B i}$. The uplink signal is $x_{M i} \in \mathbb{C}$. The noise at node $k$ is denoted $z_{k}$ and is assumed to be zero mean complex Gaussian. In the first phase (MA phase), BS transmits the signal

$$
\boldsymbol{w}_{B 1}\left(P_{B 1}\right)^{\frac{1}{2}} x_{B 1}+\boldsymbol{w}_{B 2}\left(P_{B 2}\right)^{\frac{1}{2}} x_{B 2} .
$$

At each SBS, the signal $y_{S i}$ is received, where

$$
\begin{aligned}
y_{S i} & =\boldsymbol{h}_{B i}^{H} \boldsymbol{w}_{B i}\left(P_{B i}\right)^{\frac{1}{2}} x_{B i}+\boldsymbol{h}_{B i}^{H} \boldsymbol{w}_{B j}\left(P_{B j}\right)^{\frac{1}{2}} x_{B j}+h_{M i}\left(P_{M i}\right)^{\frac{1}{2}} x_{M i}+z_{S i} \\
& =\boldsymbol{h}_{B i}^{H} \boldsymbol{w}_{B i}\left(P_{B i}\right)^{\frac{1}{2}} x_{B i}+h_{M i}\left(P_{M i}\right)^{\frac{1}{2}} x_{M i}+z_{S i},
\end{aligned}
$$

where $i, j=1,2, i \neq j$, and where we have used that $\boldsymbol{h}_{B i}^{H} \boldsymbol{w}_{B j}=0$ from the zero forcing.

The power in the signal from BS to SBSi is $P_{B i}\left|\boldsymbol{h}_{B i}^{H} \boldsymbol{w}_{B i}\right|^{2}$, and the noise power is $\sigma^{2}$. Because we assume that each MS transmits at rate equal to capacity of its link, the SNR of the link between MSi-i and SBSi is $\gamma_{M i}=2^{R_{U i}}-1$ The SNR between BS and SBSi is $\gamma_{B i}=\frac{P_{B i}\left|\boldsymbol{h}_{B i}^{H} \boldsymbol{w}_{B i}\right|^{2}}{\sigma^{2}}$. From this, we can determine the rate region at SBSi in the MAC phase:

$$
\begin{aligned}
R_{U i} & \leq \log _{2}\left(1+\gamma_{M i}\right) \\
R_{D i} & \leq \log _{2}\left(1+\gamma_{B i}\right) \\
R_{U i}+R_{D i} & \leq \log _{2}\left(1+\gamma_{M i}+\gamma_{B i}\right)
\end{aligned}
$$

Assuming equality in (11), since user transmits at full rate, we substitute (11) into (13). After some manipulations, we get

$$
\frac{\sigma^{2}\left(1+\gamma_{M i}\right)\left(2^{R_{D i}}-1\right)}{\left|\boldsymbol{h}_{B i}^{H} \boldsymbol{w}_{B i}\right|^{2}} \leq P_{B i}
$$

which is the condition on the transmission power of the BS for SBSi. The conditions for the second phase follow from the same arguments as the single SBS case, and that the BS uses receive zero forcing.

\section{Numerical Examples}

To evaluate the performance of WEW, we consider the setup of one BS and two SBSs, each of which serving one MS. Two cases are considered, the BS having either 2 or 3 antennas. In both cases, we show three different uplink rate requirements, and for each of these, we vary the downlink rate requirements. Each channel element is assumed to be zero-mean circulary complex symmetric Gaussian. For the case of 2 antennas, which is shown in Fig. 2, we see that for increasing downlink rates, the required minimum transmission power at the BS increases. For the uplink rates, we see that an increase by a factor of 2 requires an increase in transmission power by approximately $8 \mathrm{~dB}$. For the case of 3 antennas at the BS, shown in Fig. 3, we see the same behaviour. However, in this case, the BS has 3 antennas, which means that it has 3 degrees of freedom in performing the zero forcing. This translates into a decrease in minimal transmission power of about $10 \mathrm{~dB}$, compared to the 2 antenna case, when considering the same uplink and downlink rate requirements. 


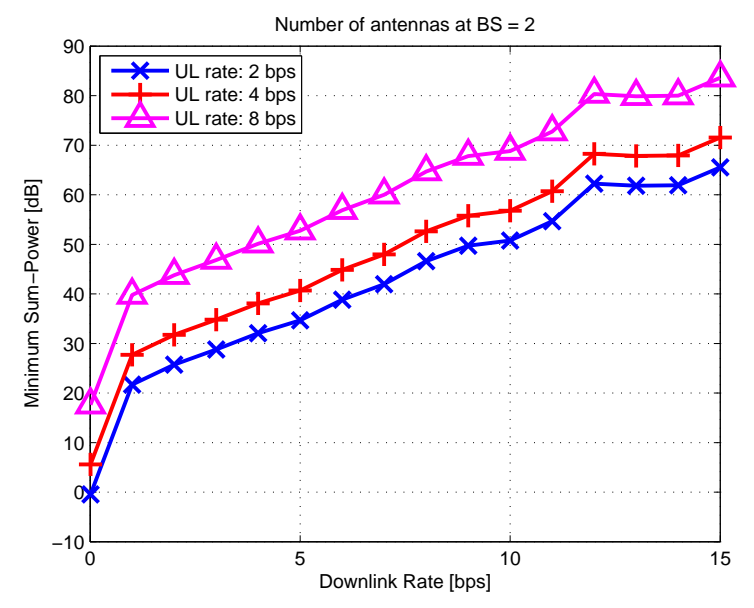

Figure 2: Case of 2 antennas at BS.

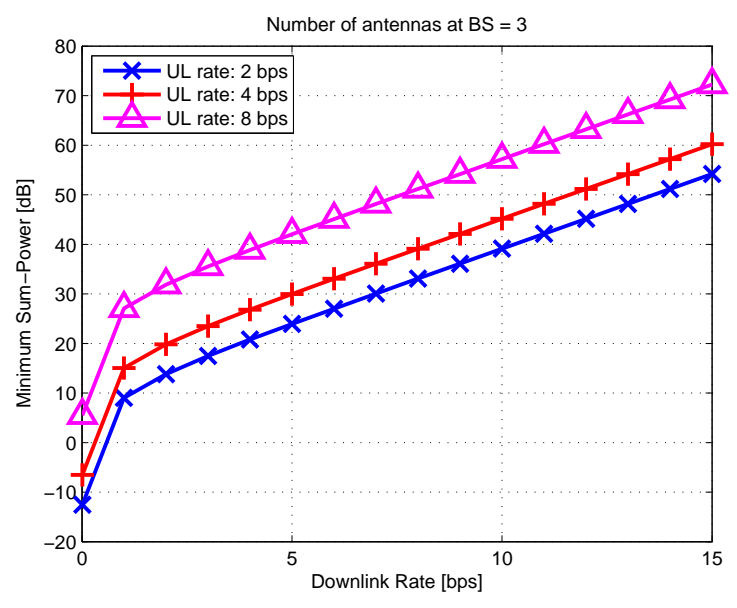

Figure 3: Case of 3 antennas at BS.

\section{Conclusion}

In this paper, we have investigated solutions for addressing the network densification challenge in next-generation wireless networks. We have proposed a wireless backhaul solution and provided conditions under which it can emulate a wired backhaul solution, in a way that is transparent, at the physical layer, for the end user. We have looked at two examples, with a single and two SBSs, respectively. For a single MS we have determined the minimal power required for emulation, while for the case of two small cells, we have used zero forcing at the BS to spatially separate the data streams. Numerical results were provided to show the relative performance in terms of transmission power for several uplink and downlink rates.

\section{Acknowledgement}

Part of this work has been performed in the framework of the FP7 project ICT-317669 METIS, which is partly funded by the European Union. The authors would like to acknowledge the contributions of their colleagues in METIS, although the views expressed are those of the authors and do not necessarily represent the project.

\section{References}

1. J. G. Andrews, H. Claussen, M. Dohler, S. Rangan, and M. C. Reed. Femtocells: Past, present, and future. Selected Areas in Communications, IEEE Journal on, 30(3):497-508, 2012.

2. N. Bhushan, Junyi Li, D. Malladi, R. Gilmore, D. Brenner, A. Damnjanovic, R. Sukhavasi, C. Patel, and S. Geirhofer. Network densification: the dominant theme for wireless evolution into 5g. Communications Magazine, IEEE, 52(2):8289, February 2014.

3. T. Brown, P. Kyritsi, and E. De Carvalho. Practical Guide to the MIMO Radio Channel: with MATLAB Examples. John Wiley \& Sons, 2012.

4. P. Popovski and H. Yomo. Bi-directional amplification of throughput in a wireless multi-hop network. In Vehicular Technology Conference, 2006. VTC 2006-Spring. IEEE 63rd, volume 2, pages 588-593. IEEE, 2006.

5. P. Popovski and H. Yomo. Wireless network coding by amplify-and-forward for bi-directional traffic flows. Communications Letters, IEEE, 11(1):16-18, 2007.

6. C.D.T. Thai and P. Popovski. Coordinated direct and relay transmission with interference cancelation in wireless systems. Communications Letters, IEEE, 15(4):416-418, 2011.

7. C.D.T. Thai, P. Popovski, M. Kaneko, and E. De Carvalho. Coordinated transmissions to direct and relayed users in wireless cellular systems. In Communications (ICC), 2011 IEEE International Conference on, pages 1-5. IEEE, 2011. 